Mots. Les langages du politique

\title{
Un Français à la cour du Morho Naba
}

\section{Olivia Guérin}

\section{OpenEdition}

Journals

Édition électronique

URL : https://journals.openedition.org/mots/762

DOI : $10.4000 /$ mots. 762

ISSN : 1960-6001

\section{Éditeur}

ENS Éditions

\section{Édition imprimée}

Date de publication : 1 novembre 2006

Pagination : 23-36

ISBN : 978-2-84788-099-1

ISSN : 0243-6450

\section{Référence électronique}

Olivia Guérin, "Un Français à la cour du Morho Naba », Mots. Les langages du politique [En ligne], 82 I 2006, mis en ligne le 01 novembre 2008, consulté le 22 avril 2022. URL : http:// journals.openedition.org/mots/762 ; DOI : https://doi.org/10.4000/mots.762 


\section{Olivia Guérin}

\section{Un Français à la cour du Morho Naba ${ }^{1}$}

Un certain nombre de travaux récents s'intéressent à l'emprunt non plus dans la perspective traditionnelle d'une sémantique lexicale (dans la lignée de Louis Deroy, 1956), mais dans le cadre d'une sémantique énonciative et discursive, où l'emprunt est analysé comme le lieu d'émergence d'un point de vue ${ }^{2}$. C'est dans cette seconde perspective que se situe notre approche de l'emprunt, qui tente plus particulièrement de mettre en relation le fonctionnement de l'emprunt avec un questionnement de type générique; nous nous intéressons en effet à un genre discursif qui se révèle particulièrement accueillant à l'égard de l'emprunt: le récit de voyage. Discours produits en situation de contact de cultures, les récits de voyage permettent en effet une riche moisson de termes empruntés aux langues locales que croise le voyageur (les dénominations locales étant réputées être les plus adéquates pour désigner les réalités exotiques), en particulier dans le domaine de la description de structures sociales et politiques qui, au premier regard, ne semblent pas correspondre aux hiérarchies existant dans la société d'origine du voyageur. Mais ce qui caractérise plus encore l'emprunt dans le récit de voyage, c'est qu'il s'inscrit dans cette dialectique du même et de l'autre propre aux discours de l'altérité culturelle : alors même qu'il semble donner la parole aux sujets culturels que le voyageur décrit, il fait très généralement de sa part l'objet d'une réappropriation, et nous en dit ainsi bien plus sur les représentations qui ont cours dans la société du voyageur que sur celles qu'il est censé décrire (Guérin, 2004). Plus particulièrement, les gloses adjointes aux emprunts opèrent une « déterritorialisation-reterritorialisation $»^{3}$ des réalités exotiques décrites et des pratiques sociales de l'Autre, en opérant de forts déplacements des significations des termes empruntés.

1. Pour faciliter la lecture, nous conservons dans le corps du texte les transcriptions que donne Albert Londres des mots empruntés au moré, et mentionnons en note des formes plus communément admises (sans marquage des tons). Nous remercions Raphaël Kaboré (Paris 3) pour son aide concernant les transcriptions du moré.

2. Voir, en particulier, les travaux effectués dans le cadre de la praxématique et d'une réflexion sur la nomination comme prise de position par Paul Siblot $(1995,1996)$, ainsi que l'ensemble des contributions présentées dans P. Siblot éd., 2005.

3. Nous empruntons ces termes à Gilles Deleuze et Félix Guattari (1991) pour rendre compte du processus consistant à détourner un objet de son lieu et de sa fonction d'origine pour le replacer dans un contexte où il se verra attribuer une valeur différente : un tel processus nous semble

Université Paris 3, Syled-Res, Olivia.Guerin@univ-paris3.fr 
La présente étude propose une analyse des déplacements de sens opérés par la glose d'emprunt. Nous étudierons ici un exemple de représentation de structures politiques coutumières d'Afrique noire: la description par Albert Londres d'une cour africaine traditionnelle, celle du Morho Naba de Ouagadougou, «Empereur des Mossi », dans Terre d'ébène (1929), qui relate un voyage effectué en 1927 dans les colonies françaises d'Afrique noire. Dans cette œuvre, A. Londres dresse un portrait au vitriol de l'AOF (Afrique-Occidentale française) et de l'AEF (Afrique-Équatoriale française) et dénonce l'exploitation coloniale qu'il a pu y observer. Mais la critique sociale est généralisée et n'épargne pas plus les colonisés que les colonisateurs. Ses flèches visent en particulier certaines institutions politiques traditionnelles. Aussi nous présente-t-il un tableau mi-amusé mi-critique de la cour du Morho Naba et de la chefferie traditionnelle mossi ${ }^{4}$. On tentera de montrer en quoi la glose d'emprunt, par les déplacements de sens qu'elle opère, constitue un observatoire privilégié des phénomènes d'émergence d'un point de vue non seulement individuel (par le recours, notamment, à l'ironie), mais aussi collectif, dans un fonctionnement interdiscursif de la glose qui donne à voir l'idéologie informant le discours du voyageur.

Après avoir tenté de restituer le contexte historique et politique dans lequel prend place cette description, nous montrerons que les gloses proposées par le journaliste-voyageur ne fournissent qu'un accès très partiel au sémantisme des emprunts et aux pratiques culturelles et politiques qui leur sont liées. Seront ensuite étudiés les différents effets de sens des gloses qui tendent à bâtir une représentation déterritorialisée de l'organisation politique traditionnelle mossi, représentation empreinte de l'idéologie coloniale dominante en ce début de $20^{\mathrm{e}}$ siècle ${ }^{5}$. C'est là tout le paradoxe du texte d'A. Londres: alors même qu'il prétend dénoncer l'exploitation coloniale et les stéréotypes en vigueur à son époque à l'égard des peuples africains, il ne peut s'empêcher de reconduire ces derniers, ce qui apparait tout particulièrement dans les gloses des titres politiques empruntés.

caractéristique du traitement discursif de l'emprunt dans le récit de voyage (voir Guérin, 2005, pour les principaux processus qui concourent à cette déterritorialisation : approximation, réduction de la polysémie, axiologisation, etc.).

4. La transcription la plus communément utilisée en français est la forme invariable mossi, correspondant en fait à la forme pluriel moose (utilisée en particulier comme ethnonyme, rendu en français par « les Mossi »); le singulier correspondant est moaaga. La langue des Mossi est le moré (moore), langue voltaïque.

5. Est convoquée ici l'idéologie coloniale dans une version faible, et non dans sa version forte telle qu'elle apparait chez les penseurs de l'idéologie racialiste, tels Gobineau ou Renan. Cette dernière postule l'existence de races nettement différenciées et hiérarchisées (la « race nègre ", " grossière » et « primitive », étant généralement placée au plus bas de l'échelle des peuples) et justifie ainsi une politique de soumission des races « inférieures ». Ce qui est sous-jacent au texte d'A. Londres, c'est l'idée d'une infériorité des peuples africains, et la conviction que les Occidentaux ont un devoir de civilisation envers ces peuples primitifs. 


\section{La cour du Morho Naba : contexte et structure hiérarchique}

Les Mossi constituent l'ethnie majoritaire de l'actuel Burkina Faso et habitent une région qu'ils appellent le Moogo ("pays mossi » et, par extension, «le monde »). Ils sont les descendants de conquérants qui imposèrent au $15^{\mathrm{e}}$ siècle un pouvoir de type monarchique à des populations autochtones d'agriculteurs, en justifiant cette emprise par une idéologie du pouvoir : leur dieu unique, Naab a Wende, leur aurait conféré le $n a a^{6}$, «cette force de Dieu qui permet à un homme d'en commander un autre » (Elliot P. Skinner, cité dans Savonnet-Guyot, 1985 , p. 30). Les Mossi fondèrent dès lors près d'une vingtaine de royaumes, qui connurent leur apogée au $18^{\mathrm{e}}$ siècle. À la tête de cette formation centralisée se trouve le chef du royaume de Ouagadougou, le Morho Naba, vis-à-vis duquel les autres royaumes, qui s'en sont séparés au fur et à mesure, gardent un lien de vassalité, avec différents degrés d'autonomie.

Les royaumes mossi connurent un déclin à partir du $19^{\mathrm{e}}$ siècle, en particulier du fait de la conquête française. Mais la structure politique traditionnelle mossi perdure au sein de l'Empire colonial français (Savonnet-Guyot, 1985), et même après l'indépendance - un Morho Naba existe toujours à Ouagadougou, avec un pouvoir essentiellement symbolique.

Ainsi, la description du palais et de la cour du « dernier roi nègre, le Morho Naba, empereur des Mossis» (Londres, 1929, p.138), constitue pour notre voyageur une sorte de passage obligé. Relatant une visite protocolaire du Morho Naba Kôm II7 au gouverneur de la colonie, l'extrait que nous allons analyser rend bien compte de cette permanence des structures politiques traditionnelles au sein de l'empire colonial français :

Le Morho Naba se rend chez le gouverneur pour lui présenter ses salutations hebdomadaires. Ses soronés (mignons) courent devant lui. Le Bindi Naba, ministre des musiciens, active l'ardeur des exécutants. Le Ouidi Naba, grand maître de la cavalerie, dirige la monture royale. Ce naba ne manque pas de travail : un grand nègre vêtu comme une autruche fait tant de simagrées que le cheval s'effraie ; c'est le Pouy Naba, chef des féticheurs. En voilà un que je devrais embaucher pour chasser mes chauvessouris! Chevauchant deux foulées derrière le souverain, vient le Tapsebo Naba, chef de guerre. Il est en selle sur une peau de panthère [...]. Précédant le grouillant cortège, le Ouagadougou Naba, préfet de police, œil de faucon [...]. Voilà le Nemdo Naba, chef de la viande ; le Larallé Naba, chargé de choisir les victimes destinées aux sépultures royales. Je me cache derrière un manguier. Le Ouedranga Naba, écuyer personnel. La cour est passée. La poussière retombe. Qui vient, piquant un brillant cent mètres? Encore un qui n'était pas prêt au moment du départ! Comme il court! Rien ne le gêne, pardi ! en dehors de son boubou. C'est le Kamoro Naba, le grand eunuque. Le feu estil au harem ? Naba Kôm n'est pas un roitelet. C'est le dernier empereur d'Afrique. Sur

6. Naam : « pouvoir, puissance de chef, autorité » (Alexandre, 1953).

7. Trente-quatrième Morho Naba (1905-1942). 
trois millions d'habitants au pays mossi, quinze cent mille le suivraient. L'étiquette de sa cour est rigoureuse [...]. Les soronés le suivent. Ce sont de jeunes garçons, de huit à quinze ans, choisis parmi les plus jolis. Ils ont, entre autres tâches, celles de lui verser à boire, de placer son coussin, sa natte, de porter son parapluie (ils pourraient bien le recouvrir), son sabre, de tenir les étriers, de précéder les visiteurs, et, les plus jeunes, de coucher au palais. Coiffés comme les femmes, ils portent comme elles aux poignets et aux chevilles les mêmes gros bracelets de cuivre. Défense leur est faite de fréquenter les femmes. Chaque année, le Pouy Naba, le chef sorcier, leur impose, ainsi qu'aux femmes, l'épreuve de la calebasse. Suivant la manière dont le visage se reflète dans l'eau, femmes et soronés sont déclarés innocents ou coupables. Les volages sont mis à mort. Nous interdisons cette épreuve, mais sommes-nous toujours là ? [...] Au coucher du soleil apparaît le Baloum Naba, grand intendant, porteur d'une calebasse. Incliné devant la porte du palais, il vient offrir une libation d'eau au gri-gri royal. (Londres, 1929, p. 148-151)

Comme l'ensemble de Terre d'ébène, cette description se caractérise par sa verve satirique et caricaturale, au service d'une critique généralisée visant colonisateurs et colonisés. Mais l'intérêt particulier de ce passage réside dans l'utilisation des gloses adjointes aux différents emprunts politiques, et en particulier au terme central de naba (naaba: «chef ») : elles mettent au jour une posture nettement interprétative de la part du voyageur. À un premier niveau, elles modifient profondément la structure hiérarchique de cette cour traditionnelle.

Ce qui caractérise en effet le système politique mossi, c'est une organisation extrêmement hiérarchisée, fondée sur la dévolution du naam, le pouvoir conféré par le dieu Wende, à tous les échelons de la société :

La figure centrale du dispositif institutionnel de pouvoir chez les Moose est le « chef » (naaba [...]), détenteur d'un « pouvoir» (naam), qui est une modalité hic et nunc du « pouvoir » en général. (Izard, 1995, p. 408)

Ainsi, l'ensemble de la société mossi est extrêmement hiérarchisée, et aux différents échelons sociaux se trouvent des «naba $»^{8}$; cette structure hiérarchique est reproduite au sein même de la cour royale de Ouagadougou, marquée par une étiquette très stricte. Or, la présentation qui est faite par $\mathrm{A}$. Londres de cette cour et de l'organisation politique correspondante ne rend pas compte de sa structuration effective.

Au sommet de l'ensemble des royaumes mossi se trouve le chef du royaume de Ouagadougou, le Morho $\mathrm{Naba}^{9}$, principal souverain des royaumes mossi, "chef du moogo », c'est-à-dire «chef du monde » pour les Mossi, « empereur des Mossi » pour le colonisateur. Il est entouré de nombreux dignitaires, qui se

8. Pour simplifier la lecture, nous maintenons la même forme au singulier et au pluriel pour le terme $n a b a / n a a b a$, mais le pluriel en moré est nanamse ou nanamba ; il en sera de même pour soroné/ sõgne, « serviteur», pl. sõgnkamba.

9. Moogo Naaba. 
répartissent en deux cercles : celui des dignitaires principaux et celui des dignitaires secondaires.

Le Morho Naba est assisté, pour gouverner, de six dignitaires principaux (dont seuls cinq sont évoqués dans le texte), qui sont généralement considérés comme des «ministres»: le Tapsebo Naba (Tãpsoaba ${ }^{10}$, littéralement «celui qui a la maîtrise des armes » d'où le « maître de la guerre »-Izard, 1970, p. 200), personnage de premier plan ayant les attributs d'un ministre de la guerre (Gomkoudougou V. Kaboré, 1966, p. 47) ; le Ouidi Naba ${ }^{11}$ (littéralement, «chef des chevaux »"12, d'où " chef de la cavalerie »), chargé en période de guerre de fournir les chevaux au Tãpsoaba; le Larallé $\mathrm{Naba}^{13}$, grand conseiller du Morho Naba, qui veille à l'application des règles coutumières (Kaboré, 1966) et chargé tout particulièrement de la surveillance des tombeaux royaux; le Baloum Naba ${ }^{14}$, qui a sous son autorité l'espace intérieur du palais; le dernier des dignitaires principaux est celui qu'A. Londres appelle tantôt le Kamoro Naba, tantôt le Kansoro $\mathrm{Naba}^{15}$, chargé de surveiller la cour, en particulier les femmes et les serviteurs du roi, et disposant par ailleurs d'un commandement provincial.

Le second cercle regroupe un nombre très important de dignitaires qui participent quotidiennement à la vie de la cour et contribuent à la célébration du culte royal ; seuls quatre sont évoqués dans le texte: le Pouy Naba ${ }^{16}$, titre que G. V. Kaboré (1966) reformule par «détecteur des crimes», mais qu'il convient de mettre en relation avec l'institution du poeere, jugement divinatoire destiné à s’assurer de la fidélité des épouses royales et de la chasteté des serviteurs; le Bindi $\mathrm{Naba}^{17}$ ("chef des tambourinaires»), qui préside à toutes les solennités royales et assure également la fonction de chroniqueur de l'Empire; le Ouedranga Naba ${ }^{18}$ ("chef des écuries »), qui s'occupe de l'entretien des chevaux du Morho Naba; et le Nemdo Naba ${ }^{19}$ ( chef de la viande $»^{20}$ ), qui répartit la viande des animaux sacrifiés pendant les cérémonies rituelles entre tous les ayants droit.

10. On remarquera que c'est le seul titre de dignitaire de cour doté de fonctions politiques à ne pas être construit avec le morphème naaba; or, A. Londres rétablit ce morphème et instaure ainsi une régularité paradigmatique là où elle ne s'applique pas.

11. Wiid-naaba.

12. Du radical wiid- : « cheval ».

13. Lagl-naaba.

14. Balm-naaba.

15. Les deux dénominations semblent renvoyer au même personnage. En effet, il existe bien un «grand eunuque » à la cour du Morho Naba, le Kamsãoog-naaba - nom proche de la transcription Kansoro Naba donnée pour un second personnage un peu plus loin dans le texte ; en revanche, nous n'avons pas trouvé mention d'un autre dignitaire portant un nom qui aurait pu être transcrit par Kamoro Naba.

16. Poe-naaba.

17. Bend-naaba, formé sur le radical bend-, de bendre (pl. benda) : «tambour» et, par extension, « musicien jouant de cet instrument» (Izard, 1970).

18. Werãng-naaba, de werãnga: « aire circulaire de stabulation des chevaux (Izard, 1995, p. 411).

19. Nemd-naaba.

20. Nemdo: «viande». 
Dans les cérémonies se déroulant au palais du Morho Naba interviennent d'autres personnalités n'ayant pas de fonction politique. Ainsi du Ouagadougou Naba ${ }^{21}$, chef local de Ouagadougou considéré qui n'a pas de fonction particulière dans la hiérarchie du royaume, mais qui intervient dans certaines cérémonies de cour en tant que chef de la terre de Ouagadougou.

Le passage évoque enfin les serviteurs du Morho Naba, les soronés ${ }^{22}$, serviteurs qui secondent le souverain dans toutes les cérémonies. Recrutés très jeunes, ils sont la propriété du Morho Naba au même titre que ses femmes, et sont contraints au respect de règles très rigoureuses (ils doivent en particulier rester vierges pendant toute la durée de leur service).

Ce parcours de la hiérarchie de cour mossi montre que l'ordre de présentation adopté par A. Londres ne rend pas compte de ses différents degrés (l'auteur ne mentionne pas la distinction entre les deux cercles du pouvoir central), et même qu'il la modifie parfois. Ceci apparait en particulier avec la reformulation proposée pour Bindi Naba, «ministre des musiciens»: ce naba, recatégorisé comme "ministre » alors qu'il est un dignitaire secondaire, est mis au même niveau hiérarchique que les dignitaires principaux, qui seuls ont des attributions comparables à celles de ministres. Ainsi, l'organisation globale de la description et les gloses d'emprunt tendent à effacer les différences hiérarchiques qui existent au sein de cette cour, voire à les infléchir. Mais bien plus, elles effacent ou déplacent les significations spécifiques à l'organisation décrite.

\section{Des gloses réductrices}

En premier lieu, les gloses qui accompagnent les emprunts au moré présentent pour caractéristique principale de fournir un accès très partiel au sens. En particulier, A. Londres a tendance à effacer la complexité des différentes fonctions de cour évoquées, puisqu'il ne rend compte que d'une partie des attributions de chacun des naba. Par exemple, le Kansoro Naba n'est pas simplement "le grand eunuque » du palais du Morho Naba, mais dispose également d'un commandement provincial, ce qu'A. Londres ne mentionne pas, effaçant ainsi la fonction à proprement parler politique de ce dignitaire pour ne conserver que la fonction d'intendance interne au palais de Ouagadougou. De telles gloses ne recouvrent que partiellement le sémantisme des emprunts qu'elles reformulent, l'énonciateur focalisant l'un ou l'autre aspect du statut évoqué.

Bien plus, elles ne donnent pas à voir les représentations sociales sousjacentes à ces différents titres. Ceci apparait tout particulièrement avec la glose du titre Nemdo Naba, "chef de la viande ». La traduction littérale semble ici

21. Waogdg-naaba.

22. Sõgne, pl. sõgnkamba. 
insuffisante à une saisie des attributions spécifiques de ce dignitaire; il peut être difficile, pour un lecteur français, de se représenter avec précision ses fonctions, en l'absence de toute indication complémentaire, dans la mesure où elles ne semblent correspondre à aucune fonction de cour connue en Occident. Semblable glose ne donne pas à voir les pratiques sociales liées aux dénominations socio-politiques propres à la société décrite.

Non seulement les titres sont coupés du système culturel dans lequel ils prennent sens, mais ils sont également extraits de leur système lexical d'origine. Nous avons indiqué plus haut que le titre de naba est formé sur le même radical que naam ("pouvoir», "autorité»), ce qu'A. Londres ne mentionne pas; il n'indique pas que la notion de chef est systématiquement associée à celle de pouvoir, de naam, conféré par le dieu Wende aux Mossi. En outre, l'autre composant de chacun des titres a lui-même du sens, et la dénomination d'ensemble est motivée. Or, si A. Londres opte parfois pour une traduction littérale du titre, cette motivation n'est pas soulignée de manière systématique. Ceci est particulièrement frappant pour le Morho Naba lui-même, littéralement "chef du monde », considéré comme l'incarnation même de la force conférée par le dieu Wende et faisant l'objet d'un véritable culte (Kaboré, 1966). Ainsi, A. Londres ne fait pas mention des représentations véhiculées par la dénomination Morho Naba, qu'il perçoit probablement comme un simple nom propre dépourvu de signification, et opte au contraire pour la reformulation habituellement donnée à ce souverain par l'Occident, à savoir « empereur des Mossi ».

\section{Une représentation déterritorialisée de la cour du Morho Naba}

Les gloses proposées par le voyageur pour rendre compte du sens des emprunts politiques ne sont pas seulement réductrices, mais opèrent aussi de fréquents déplacements de sens : les structures politiques traditionnelles du Moogo nous semblent réinterprétées dans un système de valeurs et un tissu interdiscursif qui leur sont hétérogènes.

\section{Interprétation stéréotypée et jugement}

Tout d'abord, les gloses adjointes aux emprunts sont le lieu privilégié d'émergence de stéréotypes, représentations collectives figées et simplifiées qui réduisent l'autre à une série limitées d'attributs jugés typiques, évidences partagées qui sous-tendent le discours et fonctionnent sur le mode du déjà-dit (Ruth Amossy et Anne Herschberg-Pierrot, 1997). Ces stéréotypes trouvent un terrain particulièrement propice dans les discours évoquant les contacts entre groupes humains, qui incitent à la définition des identités dans une dialectique du même et de l'autre. 
On peut le remarquer en premier lieu dans la glose adjointe au titre de Larallé $N a b a$, "chargé de choisir les victimes destinées aux sépultures royales », que nous avons évoquée plus haut. Cette glose, réductrice en ce qu'elle restreint les attributions du Larallé Naba à une seule de ses multiples fonctions, fait apparaitre de surcroit une interprétation : elle évoque des sacrifices et suggère qu'il peut s'agir de sacrifices humains par le mot victimes et par la phrase suivante: Je me cache derrière un manguier, qui laisse entendre que le spectateur a lieu de craindre pour sa vie; or, selon les études consacrées à la cour du Morho $\mathrm{Naba}$, le Larallé Naba est simplement chargé de la surveillance des tombeaux royaux. À l'occasion de cette brève évocation ressurgit donc le stéréotype, largement véhiculé tout au long du $19^{\mathrm{e}}$ siècle par le discours colonial, et en particulier par les textes des voyageurs, d'une Afrique barbare et sanguinaire pratiquant les sacrifices humains, voire l'anthropophagie.

Mais la convocation de stéréotypes apparait plus explicitement dans la représentation qui est donnée du Pouy Naba. Les deux gloses fournies pour ce titre, «chef des féticheurs » et «chef sorcier», ont pour effet de reconduire les représentations traditionnelles du discours colonisateur de l'époque, ce qui est encore accentué par la description de ce dignitaire, menée sur le ton de la dérision et du burlesque. La mise en discours du titre de Pouy Naba est typique de ce mouvement de déterritorialisation-reterritorialisation que nous tentons de mettre en lumière. En effet, le titre est coupé du système lexical et culturel dans lequel il fait sens originellement (la dénomination n'étant pas mise en relation avec l'institution divinatoire du poeere, alors qu'elle est motivée). Mais surtout, le titre est reterritorialisé, replacé dans un contexte qui véhicule des représentations radicalement différentes de celles qui ont cours chez les sujets culturels concernés par ces pratiques. On notera tout d'abord que les termes choisis pour reformuler ce titre, féticheur et sorcier, sont connotés négativement en français standard, et véhiculent des représentations stéréotypées caractéristiques du discours colonisateur qui dévalorise ce type de pratiques magiques, généralement considérées comme "primitives » et attestant la crédulité de ces peuples; le voyageur introduit donc une très nette interprétation dans la manière dont il rend compte des fonctions du Pouy Naba par l'introduction d'un jugement axiologique négatif.

Mais une telle coloration axiologique du discours apparait plus nettement encore si l'on compare entre elles les deux reformulations proposées pour ce même titre ; posées comme deux équivalents de Pouy Naba, les gloses «chef des féticheurs » et «chef sorcier » sont dès lors données pour des paraphrases l'une de l'autre, ce qui ne peut se produire que dans le discours colonisateur. On remarquera en effet qu'en français régional d'Afrique, féticheur et sorcier sont plutôt des antonymes que des synonymes, et indexent des pratiques culturelles perçues comme nettement différentes. Si sorcier est systématiquement péjoratif, ce n'est pas le cas de féticheur, qui est très majoritairement porteur 
de connotations positives : au sorcier la magie noire, les maléfices, les mauvais sorts; au féticheur la magie blanche, les fonctions de prêtre, de devin et de soigneur traditionnel ${ }^{23}$. Ainsi, en faisant de féticheur l'équivalent de sorcier, A. Londres reconduit un amalgame fait par les Occidentaux entre des fonctions qui sont le plus généralement séparées, voire opposées dans la conception des sujets culturels concernés. Ceci a pour effet de bâtir une représentation univoquement dévalorisante du Pouy Naba, dont seules les fonctions occultes, « primitives » et «barbares » sont mises en exergue : A. Londres insiste en particulier sur l'épreuve du poeere, qui aboutit à la mise à mort des femmes et des serviteurs infidèles. La remarque finale faite au sujet de cet exercice divinatoire - «Nous interdisons cette épreuve, mais sommes-nous toujours là ? " ne manque pas, elle non plus, de faire resurgir l'idéologie coloniale : «nous », Occidentaux, avons le devoir de civiliser ces peuplades primitives en interdisant de telles pratiques superstitieuses et barbares; mais que les représentants de l'autorité coloniale et de la « civilisation » tournent le dos, et le naturel revient au galop.

Le chef suprême des Mossi lui-même n'est pas à l'abri des sarcasmes de l'auteur; la dérision apparait dans le passage qui introduit pour le souverain une seconde dénomination: "Sa Majesté est sous le parapluie, un parapluie de coton dont deux baleines percent le dôme. Elle s'appelle Naba Kôm, “chef de l'eau", et Elle craint la pluie!»

Naba Kôm ${ }^{24}$ n'est pas en réalité le titre générique correspondant à la fonction de souverain, mais un nom de règne. En effet, dès son intronisation, un nouveau Morho Naba ne doit plus être appelé par son vrai nom ; il se choisit une devise, en rapport avec sa personnalité et ses dispositions, et se fera désormais appeler par le surnom symbolique issu de cette devise ; notre Morho Naba a choisi une devise ayant pour mot-thème koom : « eau ». A. Londres propose pour Naba Kôm la reformulation «chef de l'eau »; or, la traduction exacte de cette dénomination serait plutôt « chef eau » (en raison de l'ordre déterminantdéterminé du moré, koom, pour être déterminant, aurait dû être antéposé à $n a b a$; il est donc ici en apposition à celui-ci ${ }^{25}$ ). Le nom de règne du Morho Naba fait ainsi l'objet d'une réappropriation de la part de l'énonciateur : elle est pour lui l'occasion d'exercer sa verve ironique aux dépens du Morho Naba (ce dernier, censé commander à la pluie, puisqu'il est supposé être «chef de l'eau », ne devrait pas avoir besoin de parapluie). La glose d'emprunt ne se contente pas d'être une traduction littérale, elle est mise au service du persiflage.

De manière plus générale, c'est la structure globale de cette cour qui fait

23. Voir, par exemple, chez Karine Boucher et Suzanne Lafage (2000), les articles féticheur et sorcier, tant pour les définitions que pour les extraits de discours attestés soulignant bien cette répartition des emplois.

24. Naab a Koom.

25. Nous remercions Raphaël Kaboré pour cette remarque. 
l'objet des sarcasmes d'A. Londres. Ce qui semble se lire derrière cette accumulation de fonctions présentées comme pittoresques ou dérisoires, c'est l'inanité d'une telle organisation politique, qui multiplie les fonctions en apparence insignifiantes. Se rendant en Afrique pour dénoncer les méfaits du colonialisme, A. Londres ne peut s'empêcher de juger également les Africains et leurs structures socio-politiques traditionnelles, et de reconduire les représentations stéréotypées qui circulent sur leur compte dans sa société d’origine.

\section{Superposition de représentations hétérogènes}

Mais le phénomène qui nous semble le plus caractéristique de ce texte est celui de la superposition, par l'intermédiaire des gloses, de représentations culturelles qui sont hétérogènes les unes aux autres: les pratiques de l'autre sont relues à travers des représentations elles-mêmes très « territorialisées », spécifiques à l'Occident, voire à la France.

Nous avons souligné la glose donnée pour le titre Morho Naba, qui s'éloigne d'une traduction littérale. En optant pour la reformulation empereur des Mossi, A. Londres réinterprète l'organisation politique qu'il tente de décrire à travers les discours qui ont été tenus par l'Occident, par le colonisateur, sur les organisations politiques traditionnelles africaines : le voyageur superpose une structure politique connue (empereur, par rapport à roi) aux structures étrangères qu'il décrit pour ses lecteurs français, et qui n'y sont pas strictement réductibles; il semble en effet que le terme d'empire ne convienne pas ${ }^{26}$ pour désigner ce qui constitue plutôt une conjonction de divers royaumes répondant à une organisation de type féodal, dont les différentes composantes prêtent allégeance au royaume de Ouagadougou, tout en maintenant une certaine autonomie à son égard.

Une telle reterritorialisation apparait plus nette encore avec la glose de Ouidi $N a b a$, "grand chef de la cavalerie », qui renvoie à une référence historique très située et semble s'appuyer sur une mémoire interdiscursive. En effet, le titre de maitre de la cavalerie est spécifique à la Rome antique - le magister equitum est un magistrat exceptionnel, nommé directement par le dictateur dont il devient le principal auxiliaire ${ }^{27}$. Une telle glose permet, certes, de ramener la fonction coutumière décrite à des références politiques et culturelles plus familières au lecteur français ; mais, ce faisant, elle opère une superposition de représentations qui sont hétérogènes l'une à l'autre.

La glose de Ouagadougou Naba en «préfet de police » et « œil de faucon » ne manque pas de surprendre, et nous n'avons pas trouvé d'informations qui attesteraient des fonctions similaires à celles d’un «préfet de police » pour

26. Voir le débat récent sur l'affirmation «L'empire mossi n’a jamais existé », http://www.fr.allafrica. com/stories/200308260761.html

27. Trésor de la langue française. 
le Ouagadougou Naba. Ce qui nous semble intéressant ici, c'est que le statut évoqué est réinterprété à la lumière de l'organisation très territorialisée de l'administration française et, plus largement, à travers des représentations culturelles occidentales.

Si les informations fournies sur les serviteurs du Morho Naba paraissent dans l'ensemble conformes à la réalité historique (concernant leur habillement, leur parure, leur statut, leurs fonctions), on remarquera que la glose de soroné par «mignon» est elle aussi le lieu d'une interprétation et de l'émergence d'un point de vue. En effet, le terme mignon ne manque pas, pour le lecteur français, d'évoquer un contexte historique très spécifique : il fait référence aux favoris du roi Henri III, à qui l'on a souvent reproché leurs manières efféminées et leur homosexualité supposée. Il devient dès lors inévitable que les représentations liées au contexte de la cour d'Henri III se superposent à celles qui sont construites dans le texte, et que les discours tenus sur les favoris du roi de France soient réactivés au sein du discours tenu sur la cour du Morho Naba, notamment en ce qui concerne les relations homosexuelles des «mignons» avec le souverain - on ne manquera pas de noter que les soronés sont systématiquement comparés aux femmes du roi, tant du point de vue de leur parure ou de leur coiffure, que de celui de leur obligation de chasteté et de leur soumission obligatoire à l'épreuve du poeere. Les pratiques mossi sont ainsi décrites avec des mots qui trainent une mémoire discursive très forte - alors que le statut et les attributions de subalternes des soronés ne sont aucunement comparables à ceux des mignons d'Henri III, nobles d'épée dont le roi s'entourait et à qui il demandait éventuellement conseil ${ }^{28}$.

Le voyageur rapatrie donc les structures politiques africaines traditionnelles vers les représentations qui ont cours dans sa société d'origine, bien que ces institutions ne soient pas entièrement assimilables aux structures occidentales.

Ce passage nous semble constituer un exemple topique du fonctionnement discursif de l'emprunt dans le récit de voyage : si ce procédé de nomination semble, au premier chef, permettre de rendre compte des caractéristiques sociopolitiques de la société décrite, la manière dont est construit en discours l'accès au sémantisme des termes empruntés tend à déterritorialiser les concepts et représentations qui leur sont liés. En effet, le voyageur dépeint les pratiques de l'autre non seulement de manière fragmentaire, mais encore à travers le prisme

28. On notera également que ressurgit à l'occasion de cette évocation des soronés, mais aussi de celle de l'épreuve du poeere destinée également aux femmes du Morho Naba, l'un des lieux communs de la littérature de voyage, qui lie fréquemment exotisme et érotisme (voir Tzvetan Todorov, 1989) ; les pratiques polygames et homosexuelles attribuées au souverain constituent une figure de l'altérité fondamentale, que le voyageur se doit de rapporter dans son récit. 
de son propre univers culturel. Les gloses d'A. Londres coupent les termes empruntés de leur système lexical d'origine, font l'impasse sur les significations sociales des pratiques correspondantes ou les déplacent fortement. La structure de la cour du Morho Naba est réinterprétée au sein d'un système réducteur et dévalorisant; pris dans le réseau des discours tenus sur l'Afrique, le voyageur tend à plaquer sur les faits sociaux et politiques qu'il décrit des représentations hétérogènes. L'un des enjeux de l'analyse des processus à l'œuvre dans les discours dits « exotiques » est, dès lors, de saisir quels gauchissements sont à la source des représentations que l'on peut se faire, en Occident, des structures socio-politiques étrangères. Le texte d'A. Londres présente à cet égard un intérêt particulier, qui tient à son positionnement paradoxal : tout en prétendant se démarquer de l'idéologie coloniale, il reconduit les stéréotypes qu'elle véhicule au sujet des peuples africains ; il ne manque pas en effet de présenter les Mossi comme une population crédule, pénétrée de croyances pittoresques, entre magie et barbarie, soumise à un roitelet exotique entouré d'un nombre invraisemblable de dignitaires aux fonctions dérisoires, et à qui il est en fin de compte justifié d'apporter les bienfaits de la civilisation.

\section{Références}

ALEXANDRE P., 1953, La langue moré, Dakar, Ifan.

AMOSSYR., HERSCHBERG-PIERROTA., 1997, Stéréotypes et clichés. Langue, discours, société, Paris, Armand Colin.

BOUCHER K., LAFAGE S., 2000, Le lexique français du Gabon : entre tradition et modernité, Nice, ILF-CNRS, «Le français en Afrique », $\mathrm{n}^{\circ} 14$.

DELEUZE G., GUATTARI F., 1991, Qu'est-ce que la philosophie ?, Paris, Minuit.

DEROY L., 1956, L'emprunt linguistique, Paris, Belles Lettres.

GUÉRIN O., 2004, "Prendre la parole de l'autre. Paradoxes dialogiques de la nomination dans les récits de voyage », A. Cassanas et al. éd., Dialogisme et nomination, Montpellier, Publications de l'Université Montpellier 3, p. 215-227.

- 2005, "De bamboula à wasamba: emprunt et déterritorialisation des pratiques sociales de l'autre dans le récit de voyage ", Cahiers de praxématique, $\mathrm{n}^{\circ} 44$.

IZARD M., 1970, Introduction à l'histoire des royaumes mossi, Paris, CNRS, Ouagadougou, Centre voltaïque de la recherche scientifique, «Recherches voltaïques », n 12-13.

—1995, «Une trifonctionnalité africaine? Représentations de la souveraineté et catégories sociofonctionnelles dans le Yatenga », Cahiers des sciences humaines, $\mathrm{n}^{\circ}$ 31-2, p. 407-425.

KABORÉ G.V., 1966, Organisation politique traditionnelle et évolution politique des Mossi de Ouagadougou, Paris, CNRS, « Recherches Voltaïques », $\mathrm{n}^{\circ} 5$.

LONDRES A., 2000 [1929], Terre d'ébène, Paris, Le Serpent à plumes.

SAVONNET-GUYOT C., 1985, "Le Prince et le Naaba », Politique africaine, n² 20, p. 29-43. 
SIBLOT P., 1995, «Comme son nom l'indique ». Nomination et production de sens, thèse de doctorat, Montpellier, Université Montpellier 3.

—1996, «De la fabrique du sens: entre prototypicalité lexicale et stéréotypie discursive », S. Moirand éd., Le discours : enjeux et perspectives, Hachette, Paris, p. 112-121.

— 2005, éd., "Emprunt et variation du sens», Cahiers de praxématique, n 44 (sous presse).

TODOROV T., 1989, Nous et les autres, Paris, Le Seuil. 